\title{
EL ENCUENTRO INTERSUBJETIVO Y SUS MEDIACIONES EN LA FENOMENOLOGÍA HERMENÉUTICA DE PAUL RICOEUR
}

\section{Martin Grassi*}

Resumen: El presente trabajo intenta introducir al lector en la problemática de la intersubjetividad desde la perspectiva fenomenológica-hermenéutica de Paul Ricoeur, atendiendo a la necesidad de las mediaciones simbólicas en el orden del reconocimiento. El fenómeno de la intersubjetividad será abordado aquí desde una perspectiva gnoseológica, ética, sociológica y hermenéutica, intentando desentrañar cómo es posible el reconocimiento mutuo entre subjetividades extrañas en un mundo común. Una reflexión sobre la intersubjetividad debe tener siempre presente la irreductibilidad de la persona como tal, reconocimiento que es posible, en última instancia, por el amor y la caridad, el cual se manifiesta en la dinámica del intercambio de dones. Por otra parte, esta irreductibilidad de la persona supone el rechazo de la postulación de una tercera sustancia diferente del yo y del tú, que los subsumiera en su entidad, tal como parece suceder en el estructuralismo y en el hegelianismo. Es nuestra intención mostrar que se trata en Ricoeur de una fenomenología genética que rastrea lo extraño desde lo propio, teniendo como núcleo pivotante de su reflexión el bomo capax que somos cada uno de nosotros y que actúa siempre en un mundo compartido (Lebenswelt).

Palabras clave: Ricoeur, Intersubjetividad, Fenomenología Hermenéutica, Mediación simbólica.

Abstract: The present work pretends to be an introduction to the problem of intersubjectivity from the phenomenological and hermeneutical point of view of Paul Ricoeur. The phenomenon of intersubjectivity will be studied from a gnoseological, ethical, sociological and hermeneutical perspective, trying to disclose how a mutual acknowledgment between strange subjects in a common

Profesor y Licenciado en Filosofía (Universidad Católica Argentina). Doctorando en Filosofía (Universidad de Buenos Aires/CONICET). Profesor Adjunto de Historia de la Filosofía Contemporánea (Universidad del Salvador). Profesor Asistente de Filosofía de la Religión (Universidad Católica Argentina). Correo electrónico: martingrassi83@gmail.com 
world can be possible. Meditation upon intersubjectivity must always attend on the irreductibility of persona as such, acknowledgment made possible by love and charity, and that reveals it sense in the espirit of giveness. In the other hand, the irreducitibility of persona implies rejecting the possibility of building a third substance, different from the I and the Thou, and which would submit them to its reality, as structuralism and hegelianism seems to propose. We intend to show that Ricoeur's position is one of a genetic phenomenology that search for the strange from the own, stating the homo capax that acts always in a shared world (Lebenswelt) as its central idea.

Key words: Ricoeur, Intersubjectivity, Hermeneutical phenomenology, Symbolic mediation.

\section{El reconocimiento mutuo}

En un trabajo anterior - del cual éste conformaría su segunda y última parte-, hemos presentado la posición de Paul Ricoeur respecto a la intersubjetividad y el doble acceso que tenemos a ese fenómeno: el acceso gnoseológico y el acceso ético. ${ }^{1}$ La cuestión que ahora debemos abordar es cómo pueden ser articulados in concreto ambos accesos. No es éste un problema menor, puesto que se trata, en última instancia, de una dialéctica entre la mediación y lo inmediato: mientras que la posición del otro como obligante respecto a mis actos prácticos pareciera ser de carácter inmediato ( $\mathrm{y}$ aquí es notable la reflexión de Levinas), la posición del otro como un alter ego, como un ser al que reconozco una esencia común a la mía, pareciera ubicarse en el ámbito de la mediación (razón por la cual se le reprochaba a Husserl esta incapacidad para llegar al otro como tal). A su vez, lo inmediato y lo absoluto se hermanan, así como lo mediato y lo relativo, por lo cual la ética nos permite acceder al otro como absoluto, como aquél que no sólo no depende de nuestro capricho, sino como quien nos traslada directamente a su vida y nos involucra en ella hasta el

\footnotetext{
${ }^{1}$ Cfr. Grassi, Martín. "El doble acceso fenomenológico-ético a la realidad del otro en la filosofía de Paul Ricoeur", en Tópicos. Revista de Filosofía de Santa Fe, No 22, julio-diciembre 2011, pp. 141-164.
} 
punto de no poder sino responder a dicha llamada. Por otro lado, el acceso gnoseológico parte de uno mismo como sujeto cognoscente y pretende elevar un puente que nos permita alcanzar la costa frente a la nuestra, y así el otro es relativo al modo en que uno se acerca a él, al modo en que uno constituye el alter a partir del ego. Pero avancemos de a poco.

Para Ricoeur, la estructura del sí mismo es más compleja de lo que a primera vista pudiera pensarse. Como ya lo había visto bien Husserl, el ego se mundaniza y ocupa un lugar en el mundo como ente intramundano, aun cuando lo habite de un modo peculiarísimo (como sujeto constituyente). De este modo, el modo de aparecer en el mundo es un medio a partir del cual puede encontrarse el corazón mismo de la subjetividad. Pero esta mundanización no se reduce a la implicación del cuerpo físico en el cuerpo vivo, sino que implica también el habitar un mundo cultural e intersubjetivo. Como también había vislumbrado Husserl, el Lebenswelt es un mundo de hombres entre hombres, un mundo esencialmente intersubjetivo y, por tanto, cultural. El otro yo no es sólo un sujeto apresentado por su cuerpo físico, sino también un sujeto cualificado por predicados de orden social y cultural. La cuestión, entonces, será determinar debidamente, siguiendo a Ricoeur, una dialéctica entre el otro como prójimo y el otro como socio, y así, entonces, juzgar el lugar de las mediaciones en el orden intersubjetivo (que sea quizá la piedra de toque de toda reflexión en torno al otro).

Ricoeur escribe un pequeño trabajo sobre "El socio y el prójimo"2 en el que elabora, a partir de la parábola del buen samaritano, la dialéctica misma de este doble modo de ser del otro que tengo ante mí. En primer lugar, afirma que el prójimo pertenece al orden de la narración, es un hombre que deviene prójimo para un desconocido, por lo cual la parábola pasa de ser una narración a un paradigma de acción. "No hay, así, una sociología del prójimo; la ciencia del prójimo está en todo caso bloqueada por una praxis del prójimo; no se es un prójimo; yo me hago prójimo de alguien". ${ }^{3}$ En segundo lugar, señala que el acontecimiento del encuentro

\footnotetext{
2 "Le socius et le prochain", en Histoire et vérité, Paris, Seuil, 2001. 3 Ibíd., p. 114.
} 
que hace presente una persona a otra solo es posible allí donde toda categoría, rol o función social es trascendida. Mientras que los hombres que no auxiliaron al herido estaban ocupados en sí mismos y sus obligaciones, el samaritano viaja sin importarle su carga social, presto a cambiar de ruta y a inventar un comportamiento imprevisto: disponible, en una palabra, para el encuentro y la presencia. Y la conducta que él inventa es la de la relación de hombre a hombre, encontrándose así en el orden del acontecimiento, sin mediación de una institución. ${ }^{4}$

Por su parte, el socius es aquél a quien me dirijo a través de su función social, por lo cual se trata aquí de una relación mediata que alcanza al hombre "en tanto que...". La historia cultural humana ha forjado poco a poco un tipo de relaciones humanas cada vez más complejas y abstractas, sin embargo, la tarea de una "teología del prójimo" es intentar captar desde el principio toda su envergadura, y para ello es necesario buscar siempre la "unidad de intención" que sostiene y anima la diversidad de mis relaciones con los otros: "es la misma caridad la que dona su sentido a la institución social y al acontecimiento del encuentro". ${ }^{5} \mathrm{La}$ oposición brutal entre comunidad y sociedad no puede ser más que un momento de la reflexión. La caridad que gobierna la historia no supone ignorar la dimensión de la justicia, que es el respeto por las instituciones y la autoridad. Por el contrario, la creencia en un Reino de Dios se vive en el dolor de las contradicciones, entre las cuales se encuentra la del debate, en nuestra vida individual y en la de los grupos, entre las relaciones "cortas", de persona a persona, y las relaciones "largas", mediadas por las instituciones. ${ }^{6}$

La oposición entre el prójimo y el socio no es más que una posibilidad de la dialéctica histórica de la caridad, pero no la más

4 Cfr. Ibíd., p. 115. Debemos retomar luego nuevamente la cuestión de la mediación institucional en el orden de la intersubjetividad, pues en rigor no es posible para la hermenéutica ningún tipo de encuentro o acontecimiento sin la mediación de una institución.

${ }^{5}$ Ibíd., p. 119.

"Cfr. Ricoeur, Paul. "Fenomenología y hermenéutica", en: Del texto a la acción. Buenos Aires: Fondo de Cultura Económica, 2006, p. 47. 
significativa. Hay un maleficio propio de la institución que consiste en entenderla como toda forma organizada de lo social, objeto propio de la sociología: se trata del maleficio de la objetivación, que tanto ataca al trabajo como a organismos de la justicia distributiva, infectados por un "funcionamiento abstracto". Al fin y al cabo, toda institución tiende a desarrollar estas pasiones de poder con los hombres de los cuales disponen como si se trataran de una cierta cosa útil. De aquí que "el tema del prójimo sea desde el principio un llamado a la toma de conciencia". ${ }^{7}$ El sentido del prójimo es una invitación a situar exactamente el mal en estas pasiones específicas que se atienen al uso humano de los instrumentos: la aventura técnica, social y política del hombre no puede recibir un límite a su extensión, por lo cual el tema del prójimo no denuncia ninguna desmesura en este plano de horizontalidad, propio de dicha aventura. Su denuncia se dirige, en cambio, a la desmesura vertical, es decir, a la tendencia de los organismos sociales a absorber y a agotar en sus niveles toda la riqueza y problemática de las relaciones interhumanas. De allí que la desmesura de lo social resida en la objetivación del hombre en las relaciones abstractas y anónimas de la vida económica, social y política. En este sentido, lo social tiende a obturar el acceso a lo personal y a esconder el misterio de las relaciones entre los hombres, así como también a disimular el movimiento mismo de la caridad que anima a todos los hombres. "El tema del prójimo opera entonces la crítica permanente al lazo social: en la medida del amor al prójimo, el lazo social no es nunca lo bastante íntimo, ni lo bastante vasto. El prójimo levanta la doble exigencia de la proximidad y de la lejanía". ${ }^{8}$

Sin embargo, tampoco hay que olvidar que las relaciones son presa de las pasiones, y que la verdadera caridad se opone tanto a la justicia "inhumana" como a la caridad hipócrita. El sentido final de las instituciones es el servicio a las personas, y la caridad se esconde tras este servicio "abstracto". Así, el criterio para juzgar el valor de las relaciones

\footnotetext{
7 "Le socius et le prochain", p. 123.

${ }^{8}$ Ibíd., p. 125.
} 
humanas no pasa por si se realizan por la vía "corta" o por la "larga", sino por si se atienen o no a las personas. ${ }^{9}$

La cuestión central, por un lado, de este pequeño y rico ensayo de Ricoeur se encuentra en la afirmación de que el encuentro interpersonal es motivado por la caridad o el amor, algo que ya estaba muy presente en las reflexiones del personalismo y de Gabriel Marcel en especial. El encontrarse disponible, el estado de disponibilidad, es la condición de posibilidad de toda presencia y encuentro. Pero, a su vez, y extendiendo la reflexión existencial hacia ámbitos más amplios, la autenticidad del encuentro interpersonal no se reduce al encuentro entre un yo y un tú, sino que se extiende también a la relación yo-él. Esto que para Martin Buber y Gabriel Marcel no era posible, puesto que implicaba la necesaria objetivación de lo personal en la tercera persona, es para Ricoeur la piedra de toque para fundamentar la legitimidad ética y existencial del socius. En efecto, como hemos expuesto, es la misma caridad, el mismo amor, el que anima tanto la relación del yo-tú como la del yo-él, a pesar de las claras diferencias que se establecen entre ambos tipos de relación. ${ }^{10}$ Por otro lado, es central la afirmación de que el orden institucional no atenta

9 "De hecho entonces diré alternativamente: es la historia -y su dialéctica del prójimo y del socio- la que mantiene la envergadura de la caridad; pero es finalmente la caridad la que gobierna la relación al socius y la relación al prójimo, donándoles una común intención. Así, la teología de la caridad no parecería tener menor extensión que la teología de la historia" (Ibíd., p. 127).

10 En este sentido, Ricoeur afirma que el fondo anónimo sobre el cual se desarrolla las relaciones interpersonales no es necesariamente negativo: "La relación personal e interpersonal se desarrolla sobre un fondo de anonimato que no es forzosamente negativo. En una entrevista reciente de una radio parisina, donde se me preguntaba qué era para mí el personalismo, respondí que es la utopía (en un sentido muy positivo) donde la sociedad sería una persona de personas. Esto no sucederá jamás. Ahora bien, y es la respuesta a vuestra pregunta, la masificación es la sociedad en la cual no hay ninguna relación interpersonal, en la que todo es institución pura, inhumana, sin rostro ("Intersubjectivité, socialité, religion" (entretien avec Paul Ricoeur par Attilio Danese), en Notes et Documents, Dossier: La retour de la philosophie practique, Avril-juin 1986, p. 77). 
El encuentro intersubjetivo y sus mediaciones en la Fenomenología Hermenéutica de Paul Ricoeur - 139

necesariamente contra la afirmación de lo personal, siendo la afirmación de lo personal el criterio para juzgar el valor de las instituciones mismas.

Sin embargo, es aquí donde surge una objeción traída desde el ámbito de la sociología que pretende desarmar esta tesis y proclamarla ilusoria o falsa: en el orden comunitario, mediado por las instituciones, no es el amor el que anima las relaciones intersubjetivas, sino la conveniencia y la necesidad del otro para la propia subsistencia. De este modo, no solo peligraría el estatuto personal del socius, sino que también pareciera ponerse en jaque la dignidad personal del prójimo, puesto que todo prójimo no deja de ser una parte útil al servicio de una comunidad. ${ }^{11}$ En efecto, en el tercer estudio de su obra Caminos del reconocimiento, titulado "El reconocimiento mutuo", ${ }^{12}$ Ricoeur establece una dialéctica entre la mutualidad y la reciprocidad que busca esclarecer y superar la reducción de lo interpersonal al ámbito del dominio y de la utilidad. Supuesta la densidad ontológica del otro y del sí mismo, será necesario encontrar las condiciones de posibilidad de un auténtico reconocimiento mutuo, es decir, de un reconocimiento que sea animado no por una pasión instrumental que reduzca la persona a un objeto, sino por una afirmación de lo personal motivada por la caridad o el amor, afirmación que nos llevaba a leer en el otro -ya sea "cercano" o "lejano"- un ser digno de ser amado, o aún más

11 Resulta interesante señalar aquí dos puntos que desarrollaremos pertinentemente luego. El primero corresponde al correlato entre la extensión del amor que anima tanto la relación yo-tú como la del yo-él, como a la extensión de la objetivación del ser personal, que alcanza también ambos tipos de relación. Paradójicamente, de alguna manera, gracias al amor, todas las relaciones interpersonales -sean "cercanas" o "lejanas", sean al modo del prójimo o al del socius- son iluminadas desde el paradigma del yo-tú, mientras que gracias a la objetivación, son todas iluminadas desde el paradigma yo-él. Así, esta objeción que parte de la sociología -lo cual no es azaroso (baste recordar el "maleficio de objetividad" que denuncia Ricoeur)- seguramente implique la postura filosófica por la cual las relaciones intersubjetivas son siempre motivadas por el conflicto, tal como propone, por ejemplo, Jean-Paul Sartre, entre otros. El segundo punto corresponde a la necesaria pertenencia del prójimo a una comunidad de vida, a partir de la cual puede darse el encuentro íntimo.

12 Ricoeur, Paul, Caminos del reconocimiento, México, FCE, 2006, pp. 191-308. 
un ser que nos interpela y por el cual debemos responder. Por esta razón, esta posibilidad de un encuentro es puesta en entredicho por la primacía del interés sobre el don. ${ }^{13}$

Para el etnólogo y sociólogo Marcel Mauss, el intercambio de dones en algunas poblaciones, como la maorí de Nueva Zelanda, es un tipo especial de intercambio mercantil cuya fuerza de obligación se encuentra en el dar a cambio, en el devolver, fuerza impresa en la cosa dada misma. Así, adopta la conceptualidad latente vinculada por la tradición maorí al término hau para designar esa fuerza que, en el don, obliga a la retribución. Por su parte, Lévi-Strauss critica a Mauss por haber permanecido dentro de la mentalidad maorí y no haber tomado la distancia racional que permite desligar la fuerza de obligación a la retribución desde el objeto mismo a las reglas del pensamiento simbólico, de donde ha recibido el objeto dicha fuerza a los ojos de los maoríes. A su vez, Claude Lefort reprocha a Lévi-Strauss el ignorar la intención inmanente a las conductas de los protagonistas del intercambio de dones, al intentar reducir lo social a un universo de reglas calculables, con lo cual se elimina la significación misma del don. Coincidirán con esta crítica de Lefort aquellos que pertenecen a una sociología de la acción (opuesta a una del hecho social, propia de la tradición durkheimiana) inspirada por la fenomenología de Merleau-Ponty, que evitarán sacrificar las justificaciones de los actores en pos de las construcciones de un observador exterior.

Lo que parece claro es que, de abandonar el plano de la sociología de la acción, nos exponemos no sólo a ignorar como válidas las justificaciones dadas por los actores, sino también a la imposibilidad de oponer las dos "competencias" del amor y de la justicia. En efecto, de mantenernos en esta lógica de la reciprocidad por la cual a todo don le es obligado un contra-don al modo de la equivalencia, y así sumir el don en la categoría de lo mercantil o de una relación jurídica o equitativa, el amor

13 "Al dar y retribuir, los hombres se confirman unos a otros su subjetividad, es decir, corroboran que no son cosas" (Walton, Roberto Juan. "La fenomenología del don y la dialéctica entre disimetría y mutualidad", en Revista Latinoamericana de Filosofia, Vol. XXXII, Nº 2 (Primavera 2006), p. 332). 
El encuentro intersubjetivo y sus mediaciones en la Fenomenología Hermenéutica de Paul Ricoeur - 141

mismo, el ágape, se pierde de vista, al menos hasta que aparezca una fenomenología de la mutualidad que lo reivindique. Es central, afirma Ricoeur, señalar la diferencia entre mutualidad y reciprocidad, como presuposición fundamental de la tesis centrada en la idea de reconocimiento mutuo simbólico. Mientras que el término de "mutualidad" va a ser reservada para los intercambios entre individuos, el de "reciprocidad" será reservada para las relaciones sistemáticas cuyos vínculos de mutualidad no son más que figuras elementales de la reciprocidad. Siguiendo la propuesta de Marcel Henaff, Ricoeur intenta resolver el "enigma del don recíproco ceremonial" mediante el recurso a la idea de reconocimiento simbólico mutuo, por lo cual se demora en la discusión abierta por Mauss para incardinarla en la reflexión acerca de la categoría de lo sin precio, la cual permite separar las prácticas del don de las prácticas propias de la esfera económica, abandonando la idea de que el don es una forma arcaica del intercambio mercantil y llevando esta práctica arcaica al plano de su carácter ceremonial vinculado a su carácter simbólico. En efecto, Henaff propone una revolución de pensamiento respecto a las propuestas estructuralistas de Mauss y de Lévi-Strauss en tanto que desplaza el énfasis de la cosa intercambiada a la relación entre el donante y el donatario, y en tanto busca la clave del enigma en la mutualidad misma del intercambio entre protagonistas, llamando reconocimiento mutuo a esta operación compartida. Así, la fuerza que supuestamente residía en el objeto intercambiado, y que establecía este double bind, desaparece si se considera a la cosa dada y devuelta como la prenda del compromiso del donante en el don y el sustituto de la confianza en la aparición del gesto de reciprocidad, por lo cual es la cualidad de la relación de reconocimiento la que confiere significación a todo lo que se considera como don. Aún más, la relación de mutualidad puede considerarse como un reconocimiento que no se reconoce a sí mismo (al encontrarse enraizado en el gesto más que en las palabras) sino simbolizándose en el regalo. 
Estamos aquí, entonces, ante análisis ideal-típico, en el sentido de Max Weber, ${ }^{14}$ de la relación de mutualidad simbolizada en el don, que se constituye en el doble juego de la precisión conceptual y la ejemplificación empírica. Es clara la diferencia históricamente presente entre el don y el bien mercantil, es decir, entre lo "sin precio" y el bien mercantil. De allí que sea conveniente proceder a un examen más próximo de las experiencias concretas de la diferencia de sentido y de intención que queda entre el intercambio de dones y el intercambio mercantil, aun cuando no aparezcan opuestos sino imbricados en la práctica cotidiana enmarcada en una época determinada. De este análisis histórico surgen dificultades que corrigen la propuesta de Henaff en tanto que la relación de mutualidad instaurada por el carácter simbólico del don puede degenerarse en una relación de tipo mercantil. Por ello, Ricoeur apela a una tarea crítica que distinga entre una buena y una mala reciprocidad en orden a proteger esta experiencia efectiva de reconocimiento mutuo, apelando a los recursos normativos del análisis ideal-típico. En este sentido, Ricoeur hace hincapié ante todo, como lo hacía Claude Lefort, en el gesto mismo de dar en tanto primer gesto de relación, es decir, en su cualidad de propuesta cristalizada en la pregunta ¿por qué dar?

El compromiso en el don constituye el gesto que inicia todo el proceso. La generosidad del don suscita no una restitución, que, en el sentido propio, anularía el primer don, sino algo como la respuesta a un ofrecimiento. En última instancia, se debe considerar el primer don como el modelo del segundo don, y pensar, si se puede hablar así, el segundo don como una especie de segundo primer don. La obligación de devolver, reinterpretada por la lógica de la reciprocidad en términos de double bind, sigue siendo con mucho una construcción de débil tenor fenomenológico que da pretexto para la distinción de los dos niveles, el de las prácticas y el del círculo autónomo dotado de autotrascendencia. La fascinación ejercida por el enigma del retorno conduce a descuidar rasgos importantes de la práctica del don, encontrados por el camino,

${ }^{14}$ En el próximo apartado, acerca de la cuestión de las instituciones y el encuentro interpersonal, volveremos sobre la importancia que le adjudica Ricoeur a la sociología de Weber en relación a una fenomenología de la intersubjetividad. 
como ofrecer, arriesgar, aceptar y, finalmente, dar algo de sí al dar una simple cosa. ${ }^{15}$

Es aquí donde Ricoeur relaciona esta fenomenología del don con el ágape, en el que se acentúa el don sin espera de retorno. La espera que separa la oferta del primer don de su recepción -espera que puede ser indefinida-, se hace también espera de la sorpresa de un nuevo don prodigado por el otro, colocando así el segundo don en la misma categoría afectiva que el primero, y distinguiendo así el segundo don del orden de la restitución. "En lugar de obligación, hay que hablar, bajo el signo del ágape, de respuesta a una llamada nacida de la generosidad del don inicial". ${ }^{16}$ Siguiendo en la misma línea, debe recalcarse el recibir como la base misma del comportamiento del donatario respecto al don recibido $\mathrm{y}$, en tal sentido, el modo en que lo obliga a devolver el don. En este caso, bajo el signo del ágape, el recibir se transforma en un gesto de gratitud (que en lengua francesa se dice también reconocimiento), y "la gratitud aligera el peso de la obligación de devolver y orienta a ésta hacia una generosidad igual a la que suscitó el don inicial", ${ }^{17}$ con lo cual puede trazarse aquí la línea para distinguir la buena de la mala reciprocidad. Todo descansa, por consiguiente, en el medio de la trilogía dar-recibir-devolver. es en la gratitud en la que descansa el buen recibir, lo cual permite discernir la buena reciprocidad. En efecto, la gratitud ayuda a distinguirla en tanto que descompone, antes de recomponerla, la relación entre el don y el contradon en dos binomios: el dar-recibir y el recibir-devolver. El espacio que se abre entre ambos binomios es un espacio de inexactitud tanto respecto a la equivalencia de la justicia como a la de la venta y, por lo tanto, la doble inexactitud respecto al valor y respecto al plazo de tiempo. La gratitud introduce la categoría de lo "sin precio" en el intercambio de dones al valorar los presentes de un modo inconmensurable respecto a los costes mercantiles. Respecto al tiempo, a su vez, no hay una medida exacta para determinar el tiempo conveniente para devolver el don, lo cual es señal en

15 Ricouer, P., Caminos del reconocimiento, pp. 303-304.

16 Ibíd., p. 304.

${ }^{17}$ Ibíd., pp. 304-305. 
el ágape de que se trata de un gesto indiferente al retorno en el intercambio de los dones. Así, la gratitud abre y salva, a la vez, el espacio entre el binomio dar-recibir y el de recibir-devolver. Por último, las figuras del fracaso del don permiten profundizar el análisis ideal-típico del intercambio de dones propuesto en términos de una ética de la gratitud.

En cuanto a la lucha por el reconocimiento y a la incertidumbre de su realización en un ser-reconocido efectivo, el carácter ceremonial del don mantiene una relación compleja con el carácter simbólico de un reconocimiento que se ignora a sí mismo en la medida en que se enmascara y se significa en la gestualidad del intercambio. Aún más, este carácter ceremonial subraya y protege el carácter festivo del intercambio, carácter que, como en el perdón, pone en marcha una onda de irradiación y de irrigación que contribuye a la progresión de la historia hacia estados de paz. Lo festivo del don es en el plano de la gestualidad lo que el himno en el plano verbal, uniéndose así al conjunto patrocinado gramaticalmente por el optativo, ese modo que no es ni descriptivo ni normativo, trascendiendo así los límites de la justicia de la equivalencia. En consecuencia, en el intercambio de dones, los protagonistas sociales experimentan la realidad de un reconocimiento mutuo, aunque no nos sea dado por ello proponer una solución teórica a la cuestión del reconocimiento mutuo. ${ }^{18}$

${ }^{18}$ Es interesante notar aquí que la disimetría originaria entre el uno y el otro se mantiene en la mutualidad. Como señala Roberto Walton, "los dos enfoques [de la disimetría originaria, la de Husserl y la de Levinas,] son legítimos -no cabe una querella de prioridad- porque ponen de relieve, cada uno a su manera, un desequilibrio que persiste siempre en el trasfondo de la mutualidad y 'no deja de hacer aparecer la reciprocidad como una superación para siempre inacabada de la disimetría'. Ante estas dificultades, Ricoeur propone la vía inversa: integrar a la mutualidad la disimetría originaria. La disimetría muestra el carácter irremplazable de cada uno de los que participan en el intercambio de dones. De este modo protege la mutualidad contra la fusión y se mantiene la distancia y el respeto a la intimidad" (Walton, R., "La fenomenología del don y la dialéctica entre disimetría y mutualidad", p. 351). 
La experiencia del don, además de su carácter simbólico, indirecto, raro, incluso excepcional, es inseparable de su carga de conflictos potenciales vinculada a la tensión creadora entre generosidad y obligación; son estas aporías suscitadas por el análisis ideal-típico del don las que la experiencia del don aporta en su acoplamiento con la lucha por el reconocimiento. ${ }^{19}$

En todo caso, aun cuando quizá la lucha por el reconocimiento sea interminable, la experiencia de un reconocimiento efectivo en el intercambio de dones nos permite distinguir entre la motivación de la lucha por el reconocimiento y el apetito de poder, protegiéndola de la fascinación por la violencia que nos invade, y manteniendo como tarea la progresiva constitución de estados de paz. De lo contrario, de claudicar ante un reconocimiento efectivo, sería imposible evitar considerar a la lucha de las conciencias como una experiencia de aniquilación de sí y del otro.

\section{Las instituciones y el encuentro interpersonal}

Aun cuando hayamos planteado la cuestión de la intersubjetividad como el camino de reconocimiento de uno mismo y del otro, Ricoeur señala que dicho camino no puede ser transitado sin un tercer elemento, a saber, las instituciones. En efecto, la relación yo-tú no es una relación que pueda darse en abstracto, en un mundo vacío de significaciones y de tradiciones, sino que la intersubjetividad está traspasada en todo momento por un momento institucional (este elemento ya aparecía en los análisis respectivos al reconocimiento mutuo en el intercambio de dones). Claro que afirmar esta necesidad de mediación para el encuentro intersubjetivo no carece de dificultades. En efecto, si las instituciones son necesarias para el encuentro interpersonal, ¿acaso no es primera en el orden del fundamento la institución que los individuos como tales? Pero, si las instituciones son primeras en el orden del fundamento de la intersubjetividad, ¿acaso no desaparecen las subjetividades como tales,

${ }^{19}$ Ricoeur, P., Caminos del reconocimiento, p. 307. 
como individuos singulares e irreductibles? En otras palabras, ¿hasta qué punto podría hablarse de identidad y alteridad en un sistema de sumisión de toda diferencia respecto a un mundo institucionalizado?

La cuestión de las instituciones, entendida en su sentido amplio como la cuestión de un mundo histórico, comunalizado y culturalizado, abre el debate, pues, sobre la dialéctica entre el individuo y lo común. En esta dialéctica, jugará un papel central para Ricoeur la cuestión del mundo de la vida, central en la Krisis de Husserl, pero anunciada ya en los últimos parágrafos de las Meditaciones cartesianas. Así, Ricoeur contrapone a la fenomenología del espiritu de Hegel la fenomenología de la conciencia de Husserl en lo que respecta a la intersubjetividad. ${ }^{20}$ La pregunta que hace Ricoeur es: ¿puede la teoría de la intersubjetividad de Husserl ocupar el lugar de una teoría hegeliana del espíritu? Un primer argumento que ofrece Ricoeur para contestar afirmativamente apunta a que la constitución en y a partir del ego de Husserl, entendida en el sentido de explicitación (Auslegung, que significa en alemán también "exégesis") puede compararse al espíritu hegeliano, captado él mismo en el elemento de la conciencia. Aquí la zona de intersección entre ambas fenomenologías. Sin embargo, no se trata en Husserl de un idealismo subjetivo, puesto que, por un lado, es esencial en su fenomenología el trabajo de cuestionamiento retroactivo posibilitado por el hilo conductor trascendental del objeto, que va desde el objeto ya constituido a los estratos de sentido y a los niveles de síntesis de la conciencia, por lo cual no se trata en la conciencia de una creación de sentido. Por otro lado, Husserl busca evitar el peligro de solipsismo en la cuestión de la constitución del otro, que toma como hilo conductor la dirección hacia el otro yo manifiesta en la actitud natural y en el lenguaje, por lo cual el trabajo de sentido no comenzaría siquiera si el resultado de la constitución no regulara teleológicamente el movimiento de la constitución, procedimiento radicalmente ajeno a Hegel. En todo caso, se trata de transformar en tarea lo que en primer lugar es un hecho: los otros yoes, la naturaleza común y la comunidad humana. El lado positivo de

${ }^{20}$ Cf. Ricoeur, Paul. "Hegel y Husserl sobre la intersubjetividad", en Del texto a la acción: Ensayos de bermenéutica II, Buenos Aires, Fondo de Cultura Económica, 2006, pp. 259-278. 
este argumento estriba en que, si la constitución no es una creación de sentido, su estatuto epistemológico es el de la explicitación, es decir, el de desplegar el potencial de sentido de una experiencia. Ricoeur señala que esta explicitación en Husserl debería ser pensada en un sentido más dialéctico, prestando una mayor atención a las experiencias negativas, aun cuando lo negativo no ocupe sistemáticamente la totalidad de la experiencia y sea sólo la modalidad más dramática de la explicitación, razón por la cual ésta abarca a la dialéctica. La fenomenología es, así, la Auslegung del ego entendido en este sentido retrospectivo, y se manifiesta como un proceso indefinidamente continuado, trabajo del sentido del que no tengo la clave y que más bien me constituye como yo.

Un segundo argumento se enraíza en el papel de la analogía en la relación entre los egos. Éste es el principio que para Ricoeur ocupa el lugar del Geist hegeliano, y "significa que el alter ego es un ego como yo, y que esta analogía es el último e insuperable principio constituyente". ${ }^{21} \mathrm{Y}$ esta analogía, como principio trascendental, se extiende no sólo al prójimo -en tiempo, espacio, cultura, etc.-, sino a los lejanos, por lo cual gracias a ella la tercera y la segunda persona son también primeras personas y, así, análogas.

Un tercer argumento se encuentra en que la fenomenología de Husserl "se mantiene en la pretensión de no postular más que la mutualidad de los sujetos y nunca un espíritu en tanto entidad suplementaria". ${ }^{22}$ La analogía encuentra una aplicación privilegiada en tanto que abre un campo de realidades y de experiencias accesibles a descripciones empíricas, por lo cual se debe pensar, para Ricoeur, conjuntamente a Husserl y a Max Weber, puesto que la sociología comprensiva ofrece un cumplimiento empírico a aquel trascendental vacío de la analogía. Husserl, en efecto, establece tres puntos que describen la red a priori de una sociología comprensiva: primero, la constitución de lo ajeno en lo propio es reversible, recíproca, mutua, con lo cual yo mismo soy un alter ego para otros; segundo, la existencia social se basa en la

\footnotetext{
21 Ibíd., p. 269.

22 Ibíd., p. 272.
} 
constitución de una naturaleza común, presuponiendo para la comunicación de los objetos culturales la comunicación de la experiencia de las cosas naturales; tercero, esta puesta en comunidad se jerarquiza hasta constituir objetividades espirituales de grados diferentes, lo cual marca el lugar vacío para una composición dialéctica de estilo hegeliano, encontrándose en la cima de la gradación las personalidades de orden superior como el Estado y otras instituciones. Así, puede Husserl hablar de mundos culturales, adelantándose a los análisis de la Krisis dedicados al Lebenswelt. Si hay una tesis husserliana en sociología, afirma Ricoeur, "es que la analogía del ego debe ser seguida desde abajo hasta lo alto de las comunidades sin invocar nunca una entidad distinta de la interrelación de los egos"23, y tal parece ser la respuesta de Husserl a Hegel en este respecto.

Sin embargo, continúa Ricoeur, esta respuesta solo se completa en los análisis de contenido de Max Weber, por lo cual la quinta de las Meditaciones cartesianas no constituye por sí misma una descripción de la vida cultural ni una epistemología de las ciencias sociales, sino que ésta debe buscarse en la obra weberiana, Economia y Sociedad. En primer lugar, Weber plantea que la acción humana puede ser interpretada de manera comprensiva por sus agentes, y toda conducta ajena a la cuestión del sentido nos saca del dominio de la sociología comprensiva. Así, el individuo es el portador del sentido, lo cual define el individualismo metodológico de la sociología comprensiva y su decisión antihegeliana más primitiva. En segundo lugar, cae bajo la jurisdicción de la sociología comprensiva toda acción no sólo significante para el individuo, sino además orientada hacia el otro, que entra así en una modalidad de acción plural. En este nivel, la calificación de social se introduce como epíteto de la noción misma de acción, y no como sustantivo: inclusive el Estado es una co-acción, una "acción con". De allí que "es tarea de la sociología reducir la apariencia de objetividad a operaciones que hacen los hombres unos en relación con otros, es decir, agentes capaces de reasumir su motivación y de medirla según motivaciones típicas". ${ }^{24}$ En tercer lugar,

${ }^{23}$ Ibíd., p. 273.
24 Ibíd., p. 274. 
por último, Weber replica a Hegel la apariencia de objetividad de las instituciones y apunta a reducirla a la previsibilidad de un determinado curso de acción: es el carácter probabilístico de este curso de acción el que reificamos en una entidad separable. Este recurso a la probabilidad es decisivo en tanto que apunta a excluir la ilusión de la existencia de una entidad subsistente, por lo cual la empresa de Weber es una empresa sistemática de de-sustancialización de las entidades colectivas, y la epistemología crítica de la sociología, elevándose a una reflexión trascendental de tipo husserliano sobre esta sociología de primer grado, debe disolver esta ingenuidad pre-crítica.

Es la unión de Husserl y de Weber lo que permite responder de manera contundente a la posición de Hegel: Husserl aporta el sentido formal apriorístico de la analogía del ego como el trascendental que regula todas las relaciones recorridas por la sociología comprensiva y corroborando la convicción fundamental de que se encontrarán siempre sólo relaciones intersubjetivas y nunca cosas sociales, mientras que Weber aporta el contenido empírico, descripto en la grilla de los tipos ideales.

La sustitución del espíritu objetivo hegeliano por la intersubjetividad preserva, en mi opinión, los criterios mínimos de la acción humana, a saber, que se la pueda identificar mediante proyectos, intenciones, motivos de agentes capaces de imputarse a ellos mismos su acción. (...) La analogía del ego adquiere entonces valor de protesta. Significa que, por más reificadas que estén las relaciones humanas, esto define el malestar y el mal de la historia, no su constitución primordial. Si la analogía del ego es el trascendental de todas las relaciones intersubjetivas, la tarea entonces consiste en identificar teóricamente y hacer prevalecer prácticamente la semejanza del hombre, mi semejante, en todas las relaciones con mis contemporáneos, mis predecesores y mis sucesores. De ese modo, la intersubjetividad de Husserl puede ser elevada al rango de instancia crítica a la cual hasta el Geist hegeliano debe ser sometido. ${ }^{25}$

${ }^{25}$ Ibíd., p. 277-278. 
Las instituciones y el mundo de la cultura pertenecen, entonces, al mundo de la vida en tanto mundo intersubjetivo e histórico, y bajo ningún aspecto responden a una instancia superior hipostasiada e independiente de los agentes que conviven en el seno de una comunidad. Esta fuerte toma de posición por parte de la fenomenología husserliana protege al sujeto personal como centro de iniciativas y como ser libre. Sin embargo, no es menos cierto que la persona se encuentra ya en un mundo constituido culturalmente y con instituciones ya establecidas, por lo cual evitar una tercera substancia entre el yo y el tú no supone pensar la intersubjetividad al modo en que se ha pensado la subjetividad moderna, es decir, de modo a-histórico y transparente. La intersubjetividad misma está traspasada por la historia, teñida toda ella de una tradición hecha manifiesta en los objetos culturales y en las instituciones. Así, la relación interpersonal no se agota en el encuentro entre el yo y el tú, sino que tiene como suelo un mundo de la vida común, no sólo en tanto que es natural, sino también en tanto que es cultural. Sería interesante ver cómo un encuentro entre personas de culturas diversas precisa de la articulación simbólica común para llegar a tener lugar. En todo caso, el "polo él" es también una de las bases estructurales de la relación interpersonal, que estructurará, por ello, la dinámica ética y moral. ${ }^{26}$

\section{Conclusión}

Habiendo recorrido el camino transitado por Ricoeur a través de algunos de sus textos, puede apreciarse el carácter paradójico e

26 Dejaremos para otro trabajo la cuestión de la dinámica intersubjetiva marcada culturalmente desde una perspectiva ético-moral. Baste señalar que Ricouer ha dedicado varios trabajos a pensar la relación ética con el otro teniendo en consideración la dimensión institucional, a la que llama "polo-él" o "elemento neutro". Puede consultarse, sobre todo, los Estudios VII-IX de Soi-même comme une autre, y el artículo "El yo, el tú y la institución. Los fundamentos de la moral: la intención ética" (en Educación y politica: De la historia personal a la comunión de libertades, Buenos Aires, Prometeo Libros/Universidad Católica Argentina, 2009, cap. IV, pp. 69-80). 
inaprensible de la intersubjetividad como fenómeno fundamental de la existencia humana. El sí mismo no puede comprenderse si no es por el rodeo por lo otro, por la alteridad, y entre los diversos modos de ser otro, el del prójimo se revela con especial énfasis. La intersubjetividad, de este modo, no puede desligarse de la subjetividad misma. De allí que sean válidos los caminos desde el yo al tú (como en el caso de la aprehensión analogizante de Edmund Husserl), como también el camino del tú al yo (como en el caso de la epifanía del rostro de Emmanuel Levinas). Sin embargo, en el seno de esta paradoja del uno y del otro, ${ }^{27}$ Ricoeur pareciera apostar por el polo de la mismidad como punto de anclaje del fenómeno intersubjetivo.

En otro trabajo, ${ }^{28}$ hemos visto cómo, en primer lugar, para Ricoeur, el reconocimiento del otro se da fenomenológicamente a partir de la parificación entendida como una analogía de la atribución, analogía que se califica por la parificación y no por un acto intelectual que ponga en relación dos objetos (como en el caso de la analogía de la proporcionalidad), es decir, por la aprehensión del otro como un alter ego. A su vez, veíamos cómo un cierto a priori de carácter ético me revela el valor irreductible del otro en cuanto otro, antes de cualquier tipo de constitución fenomenológica. Aún en este caso, la prioridad del otro respecto a uno mismo se revela desde uno mismo al modo de un imperativo ético reconocido como un a priori de la percepción, por lo cual no se trata aquí de un planteo levinasiano.

En el presente trabajo, desde un punto de vista ético y comunitario, hemos visto cómo el otro deja de ser un mero objeto social para convertirse en un prójimo. En este caso, sigue siendo uno mismo quien toma como tarea esta responsabilidad por el otro desde el movimiento de la caridad, que nace de la estima de sí mismo para moverse hacia la estima del otro -no en el sentido de una génesis temporal, pero sí de una génesis del sentido. De aquí se sigue no la prioridad del otro como

\footnotetext{
${ }^{27}$ Cfr. Ricoeur, P., Les paradoxes de l'identité, pp. 203-204.

${ }^{28}$ Cfr. Grassi, Martín. "El doble acceso fenomenológico-ético a la realidad del otro en la filosofía de Paul Ricoeur".
} 
fenómeno fundante de sentido, sino la prioridad de la razón práctica sobre la razón teórica como condición de posibilidad del fenómeno de la alteridad, razón por la cual la lógica y la objetivación pierden al prójimo y al socio como personalidades para transformarlas en haces funcionales (y de aquí la ilusión abstracta del estructuralismo sociológico y de la reificación de lo social de sello hegeliano): mientras que la lógica pareciera sumir la intersubjetividad en el orden de la reciprocidad, la fenomenología pretende vislumbrar el horizonte de mutualidad que especifica toda relación intersubjetiva y que permite hablar de una buena y de una mala reciprocidad.

Los mismos análisis de la ipseidad desde el paradigma de la promesa parecen mostrar la tensión desde el uno hacia el otro, en tanto que el modo de la permanencia del ipse no se reduce al de la sustancialidad sino al permanecer abierto a la solicitud del otro en el compromiso asumido, por lo cual la promesa se distingue de la constancia de sí. ${ }^{29}$ Es cierto que aquí parece ser el otro quien despierta la ipseidad en el modo de la solicitud, pero nos vemos nuevamente arrastrados hacia el polo de la mismidad desde el momento en que la promesa se comprende desde la estima de sí. Debemos insistir en que esta toma de posición respecto al ego como polo definitorio del nosotros no conlleva a los ojos de Ricoeur una sumisión de la alteridad a la mismidad, sino que se trata, por el contrario, de una fenomenología genética que rastrea lo extraño desde lo propio.

La importancia del elemento neutro en la relación intersubjetiva, representada por la institución -en sentido amplio del término-, nos lleva a su vez a ubicar la ipseidad en un marco comunitario que la define como tal. De aquí que el cogito se entienda en Ricoeur como un cogito herido, como un cogito que se comprende gracias al rodeo por los símbolos. El mundo de la vida como mundo intersubjetivamente válido y constituido nos abre al nosotros como el horizonte de sentido del ego, pero esta atención a lo comunitario no tiene el sello de una fundación metafísica, sino de una fundación fenomenológica del sentido mismo del mundo

${ }^{29}$ Cfr. Ricoeur, P., Caminos del reconocimiento, Segundo estudio, III. 
circundante, y de todos los seres en él implicados -incluido el sí mismo como un otro para los otros. En ese sentido, sería interesante notar que en el reconocimiento mutuo subyace un ámbito común, el mundo de la vida, que es la condición de posibilidad misma de una efectiva comunicación y comunalización del uno con el otro. Esta cuestión es central, a su vez, para restituir las mediaciones en el ámbito de la intersubjetividad, las cuales, lejos de imposibilitar la epifanía de la alteridad, la posibilitan.

En todo caso, me parece que sería prometedor prolongar las valiosísimas indicaciones de Paul Ricoeur respecto al fenómeno intersubjetivo hacia el ámbito metafísico, abriendo la pregunta desde la génesis del sentido del uno y del otro hacia la fundamentación metafísica del uno y del otro en un nosotros fundante, abriendo el horizonte a una metafísica de la participación que, lejos de desestimar la alteridad en orden a la totalidad, pueda realizarse desde la atestación de la alteridad y de la mismidad a partir de una atestación primordial de un absoluto que los fundamente y que les done su ser. Quizá la contraposición de la primacía de la mismidad o de la primacía de la alteridad pueda encontrar una feliz reconciliación en la primacía del nosotros. Pero nos encontramos aquí más allá de la fenomenología hermenéutica de Paul Ricoeur.

Recibido: 07/2013. Aceptado: 011/2013 